\title{
Research on Ant Colony Algorithm Optimization Neural Network Weights Blind Equalization Algorithm
}

\author{
Yanxiang Geng ${ }^{1}$, Liyi Zhang ${ }^{1,2}$, Yunshan Sun ${ }^{11,2^{*}}$, Yao Zhang ${ }^{1}$, \\ Nan Yang ${ }^{1}$ and Jiawei $\mathrm{Wu}^{1}$ \\ ${ }^{1}$ College of Information and Engineering Tianjin University of Commerce, \\ Tianjin, 300134, China; ${ }^{2}$ College of Information and Engineering Tianjin \\ University, Tianjin, 300072, China \\ gengyanxiang@163.com
}

\begin{abstract}
The project of ant colony algorithm optimization neural network combining blind equalization algorithm is proposed. The better initial weights of neural networks are provided because of the randomness, ergodicity and positive feedback of the ant colony algorithm. And then, a combination of optimal weights are found through BP algorithm, which is fast local search speed. Thus blind equalization performance is improved. Computer simulation show that, the novel blind equalization algorithm speeds up the convergence rate, reduces the remaining steady-state error and bit error rate, which is compared with the Neural Network Blind Equalization Algorithm(NNBE) and Genetic Algorithm optimization Neural Network Blind Equalization Algorithm(GA-NNBE) .
\end{abstract}

Keywords: Ant colony algorithm; Neural network; Blind equalization; Initial weight; Algorithm optimization

\section{Introduction}

With the development of modern electronic information technology, a higher communication quality was required. In other way, undistorted signals are transferred in the channel. In order to eliminate and reduce the Inter-symbol Interference (ISI) caused by multipath time delay in broadband communication, a channel equalization technology was proposed to compensate channels or the whole transferring system ${ }^{[1]}$. Furthermore, it corrects the channel distortion in the receiver, and makes up for the amplitude-frequency and phase-frequency characteristic ${ }^{[2]}$. Therefore, the channel equalization has become a key technology in modern communication.

Blind equalization algorithm was widely recognized in industrial sectors such as signal processing, communication, radar detection and control and in academic circles. It enjoys a considerable development, especially with the introduction of artificial intelligence theory, which gives birth to a number of improved blind equalization algorithm, such as the blind equalization based on Constant Modulus Algorithm (CMA $)^{[3 \sim 6]}$, blind equalization algorithm of weighted multimodulus ${ }^{[7]}$, blind equalization of immune algorithm ${ }^{[8 \sim 9]}$, and a hybrid wavelet neural network blind equalization algorithm based on fuzzy control ${ }^{[10]}$.

In recent years, blind equalization algorithm of neural network has achieved fruitful results. A variety of blind equalization algorithms of feedforward ${ }^{[11 \sim 13]}$ and recursive ${ }^{[14 \sim 16]}$ neural network were developed. However, the adoption of Error Back Propagation Algorithm (BP) to train neural networks leads to two problems, namely, the slow convergence speed and easily falling into locally optimal solution. Ant colony algorithm

Yunshan Sun is the corresponding author. 
is a new bionics optimization algorithm, which achieves an indirect message delivery via the pheromone released by ants when they are in their foraging and the following ants will choose and decide the nearest route for foraging and homing according to the intensity of the pheromone ${ }^{[17]}$. The convergence ability of neural network can be improved and the training time can be shortened effectively by making use of the strong adaptation, the positive feedback and the robustness of ant colony algorithm to optimize the initial weight of the neural network. This thesis firstly optimizes the initial weight of the neural network by ant colony algorithm, which is then applied to the blind equalization algorithm and leads to the iterative formula of the algorithm. A Matlab simulation is conducted to verify the effectiveness of the algorithm.

\section{The Basic Ideas on Ant Colony Algorithm Optimizing Neural Network Weight in Blind Equalization Algorithm}

The principle diagram presented in fig. 1 shows how ant colony algorithm optimizes neural network weight in the blind equalization algorithm(ACO-NNBE). The basic idea of this algorithm is to make use of the ergodicity of ant colony algorithm to help neural network rapidly find out the better solution of the weighted combination, and conduct a fast convergence with the help of BP algorithm to avoid falling into locally optimal solution. The fast convergence also improves the ability of channel blind equalization and achieves a better equalization effect.

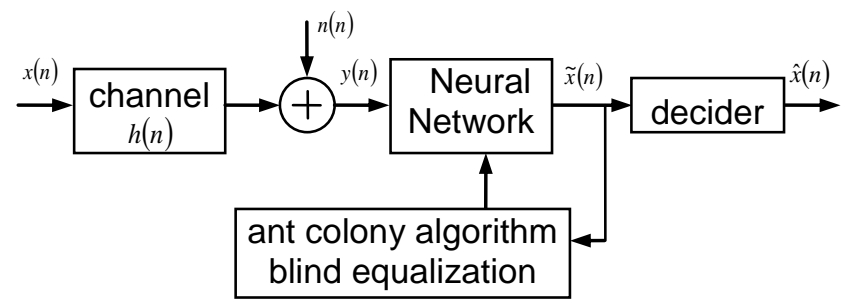

Figure 1. The Principle Diagram of Optimizing the Blind Equalization of Neural Network Weight based on Ant Colony Algorithm

In Figure 1, $x(n)$ refers to the input signal; $n(n)$ refers to the added noise of the channel; $h(n)$ represents the impulse response of the channel; $y(n)$ is the receipt signal, which is also the input signal of neural network; $\tilde{x}(n)$ is the output signal after the blind equalization and $\hat{x}(n)$ refers to the decision device output.

\section{Ant Colony Algorithm Optimizing the Blind Equalization of Neural Network Initial Weight and its Realization}

The choice of the initial weight of neural network will have influence on the final solution of training as different initial weight settings will probably affect the time and convergence performance in a significant way. Within the training number of cycles, the closer the choice of initial weight to the optimal solution, the faster the convergence speed of neural network will be. To find the appropriate initial weight, it is suggested to combine ant colony algorithm and BP neural network to discover a better initial weighted combination of neural network. Then use the BP algorithm to search for the best weight for neural network to avoid falling into locally optimal solution. The flow chart of the system is shown in Figure 2. 


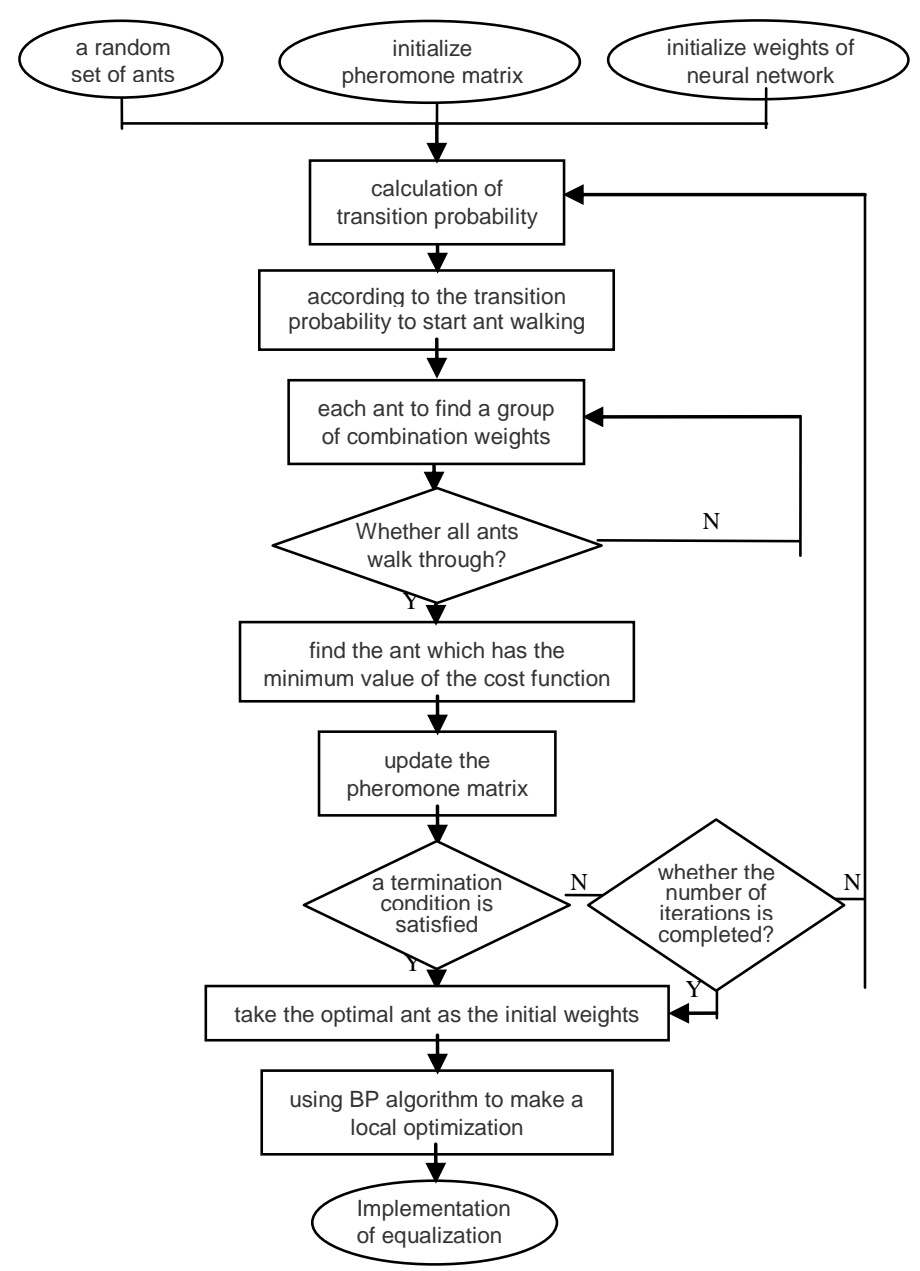

Figure 2. The Flow Chart of the System

\subsection{The Selection of Neural Network}

Firstly, select the three layers of feedforward neural network, as shown in Fig. 3:

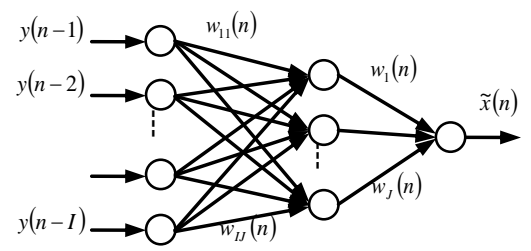

Figure 3. Three Layers of Feed Forward Neural Network

The input of neural network is $y(n-i)$, and the output is $\tilde{x}(n)$. The input of every neuron is $u$, and the output is $v$, for example, $u_{i}^{I}$ represents the input signal of the $i$ th neuron of layer $I$. The connection weight between the input layer and hidden layer is $w_{i j}(n)(i=1,2,3, \cdots, I ; j=1,2,3, \cdots, J)$, and the connection weight between the hidden layer and the output layer is $w_{j}(n)$. Therefore, the state equations of the three layers of feedforward neural network is as follows: 
(1)

$$
u_{i}^{I}(n)=y(n-i)
$$

$$
v_{i}^{I}(n)=u_{i}^{I}(n)=y(n-i)
$$

(2)

$$
u_{j}^{J}(n)=\sum_{i=1}^{I} w_{i j}(n) v_{i}^{I}(n)=\sum_{i=1}^{I} w_{i j}(n) y(n-i)
$$

$$
v_{j}^{J}(n)=f_{1}\left(u_{j}^{J}(n)\right)=f_{1}\left(\sum_{i=1}^{I} w_{i j}(n) y(n-i)\right)
$$

$$
u(n)=\sum_{j=1}^{J} w_{j}(n) v_{j}^{J}(n)
$$

$$
v(n)=\tilde{x}(n)=f_{2}(u(n))=f_{2}\left(\sum_{j=1}^{J} w_{j}(n) v_{j}^{J}(n)\right)
$$

where, $f_{1}(\cdot)$ and $f_{2}(\cdot)$ respectively refers to transfer function of the hidden layer and output layer.

\subsection{Ant Colony Algorithm Optimizing the Initial Weight of Neural Network}

The idea of ant colony algorithm optimizing the initial weight of neural network is that each ant search out a group of weighted combination within the weight solution space according to updated pheromone matrix by mean square error of the neural network output, in order to help the following ants converge to the same route, that is, the same weighted combination, which will serves as the initial weight of neural network.

3.2.1. Initializing of the Ants: To produce in random $q$ individual values within the weight range $(-b, b)$ in the neural network. Every value will produce in random a corresponding initial rates and is kept in pheromone matrix $\tau_{i j}$ as the initial pheromone density for each weight.

3.2.2. The Selection of Heuristic Functions: Blind equalization algorithm selects the weight value without the help of empirical value, therefore, there is no heuristic factor in the state transfer strategy, and the selection is conducted in random according to the probability of the pheromone density on route. According to prabability transfer formula, each ant chooses one from the $q$ individual values to serve as the weight ${ }^{w_{i j}}$ from input layer to output layer, and the weight ${ }^{w_{j}}$ from hidden layer to output layer, which will be placed in Tabu as a weighted combination of neural network. The probability transfer formula is as follows in equation 7:

$$
P\left(\tau_{i j}\right)=\frac{\tau_{i j}}{\sum_{j=1}^{q} \tau_{i j}}(j=1,2,3, \cdots, J)
$$


3.2.3. The Pheromone Updating: The pheromone updating takes place after the selection of a weighted combination by each ant and the discovery of the optimal solution, and the pheromone matrix update is accroding to the fomula 8:

$$
\tau_{i j}= \begin{cases}(1-\rho) \cdot \tau_{i j}+\rho \cdot \Delta \tau_{i j}^{(k)} & \text { if }(i, j) \text { is the } \\
& \begin{array}{l}
\text { optimal weights } \\
\text { of the iteration }
\end{array} \\
(1-\rho) \cdot \tau_{i j} & \text { other }\end{cases}
$$

Where, $(1-\rho)$ is the evaporation rate of pheromone, $\rho \in(0,1)$; and $\Delta \tau_{i j}^{(k)}$ is the changing rate of the pheromone of the weight value selected by the $k$ th ant, the value of which can be referred to as $\Delta \tau_{i j}^{(k)}=Q / L$, with $Q$ as the coefficient and $L$ as the error of this iterative optimal weighted combination.

3.2.4. The Terminal Conditions of Iterative: When using ant colony algorithm to optimize neural network initial weight, there are two terminal conditions, one is ant colony algorithm cycle number $N C$, the other is the error value less than a specific value $\beta$. In this thesis, either condition above will help end the cycle.

\subsubsection{Main Steps in Ant Colony Algorithm Optimizing Neural Network Weight:}

Step 1 The number of ant groups in initial ants is $M$, and the number of weight value is Dim, while the initial pheromone matrix refers to $\tau_{i j}$ as the route of each ant refers to a complete neural network. The iterative cycle coefficient is set to be $N C=1$.

Step 2 Launch the traveling of ants according to the formula of probability transfer. Each ant forms a set of neural network weight combination after one search, which is kept in tabu list Tabu ;

Step 3 Bring the combination found by each ant into neural network and calculate the output error $E$, and select the weight $w_{i j}$ that has the smallest error;

Step 4 Renew the pheromone matrix $\tau_{i j}$, and empty the tabu list Tabu .

Step 5 If it meets the iterative terminative condition, then the cycle will be ended. Otherwise, it will jump back to step 2 and it is set that $N C=N C+1$.

When the result of the cycle meets one of the iterative terminative conditions, the system will stop the cycle automatically, and the optimal weight combination will serve as the initial weight of neural network, which will later be trained by traditional BP algorithm to find the optimal solution.

\subsection{Ant Colony Algorithm Optimizing the Neural Network in Blind Equalization Algorithm}

Ant colony algorithm search out a group of overall better solutions for the neural network and continue its search for optimal solution based on traditional BP algorithm till it meets the need of balance. The transfer function of neural network is as follows:

$$
f(x)=x+\alpha \frac{e^{x}-e^{-x}}{e^{x}+e^{-x}}
$$

(9) 
Where, $\alpha$ is the coefficient, the size of which can be adjusted to achieve the effect of balance of the modulation signal. If the range of signals is small, a smaller $\alpha$ will be chosen, otherwise, the bigger $\alpha$ is chosen.

Cost function selects the constant modulus algorithm[18] as follows :

$$
J(n)=\frac{1}{2}\left[\left.\tilde{x}(x)\right|^{2}-R^{2}\right]^{2}
$$

Where, $\left.R=\frac{\left[x(n)^{4}\right.}{\left[x(n)^{2}\right.}\right]$. The purpose of blind equalization algorithm for neural network is to find the minimum value of the cost function.

Based on the most fast grads descent methodology, it can be elicited that the iterative formula for the weight values of all layers in the neural network is as follows:

$$
\begin{aligned}
& w_{j}(n+1)=w_{j}(n)-2 \mu_{1}\left[\tilde{x}(n)-R_{2}\right] \\
& f^{\prime}(u(n)) v_{j}^{J}(n) \\
& \left.w_{i j}(n+1)=w_{i j}(n)-\left.2 \mu_{2} \tilde{x}(n)|| \tilde{x}(n)\right|^{2}-R_{2}\right\rfloor \\
& f^{\prime}\left(u_{j}^{J}(n)\right) w_{j}(n) y(n-i)
\end{aligned}
$$

Where, both $\mu_{1}$ and $\mu_{2}$ are the iterative steps of the weight values.

\section{Experimental Simulation}

2PAM signal is adopted as the input signal, and the structure of the neural network is 7-9-1. Common channel $H_{1}(z)$ and telephone channel $H_{2}(z)$ are selected as simulation channel, whose transmission function is as follows:

$$
H_{1}(z)=1+0.5 z^{-1}+0.25 z^{-2}+0.125 z^{-3}
$$

$$
\begin{aligned}
H_{2}(z)= & 0.005+0.009 z^{-1}-0.024 z^{-2}+0.854 z^{-3} \\
& -0.218 z^{-4}+0.049 z^{-5}-0.016 z^{-6}
\end{aligned}
$$

The number of weights is $\operatorname{Dim}=72$, and the number of ants is twice the weights number, $M=144$.The maximum number of iterations is $N C=40$, and signal-to-noise ratio is $20 \mathrm{~dB}$. The evaporation rate of pheromone is $\rho=0.02$, and the iterative step is $\mu_{1}=\mu_{2}=0.02$. According to the result of the literature [18], ant colony algorithm optimizing neural network in blind equalization algorithm(ACO-NNBE) is compared with common blind equalization of neural network(NNBE) and genetic algorithm optimizing neural network in blind equalization algorithm(GA-NNBE) in the different channel, as shown in Figure 4 and Figure .5. The curve is an average value of 10 times. 


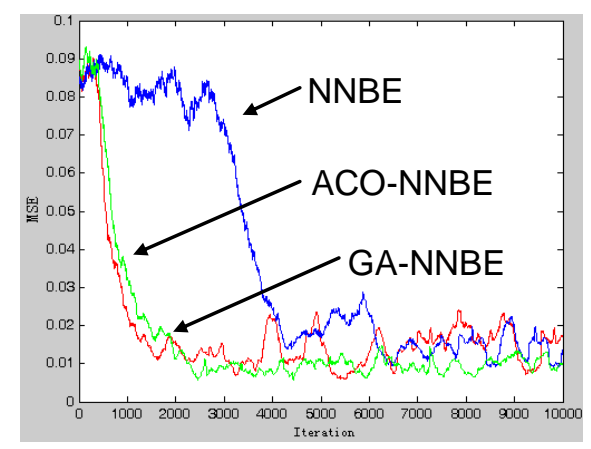

Figure 4. A Comparison of Convergence Performance in Common Channel

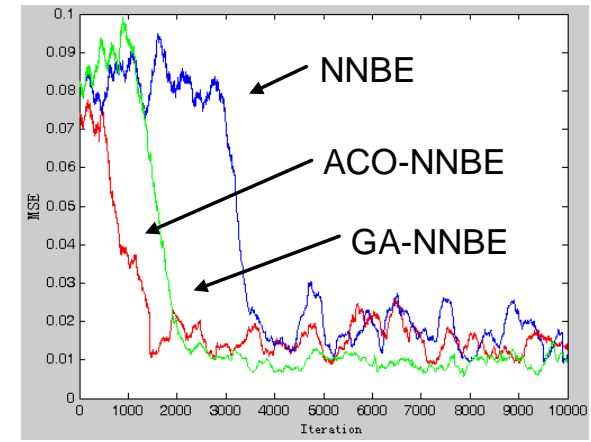

\section{Figure 5. A Comparison of Convergence Performance in Typical Telephone Channel}

It can be concluded from the two figures that in common channel both ant colony algorithm optimizing neural network in blind equalization algorithm(ACO-NNBE) and genetic algorithm optimizing neural network in blind equalization algorithm(GA-NNBE) can achieve the effect of fast convergence. The error of mean square after the convergence is not as stable as that of genetic algorithm and the speed of convergence is apparently higher than common blind equalization of neural network(NNBE). In typical telephone channel, the convergence speed of ant colony algorithm optimizing neural network in blind equalization is higher than that of neural network optimized by genetic algorithm in blind equalization, which proves the advantage of ant colony algorithm.

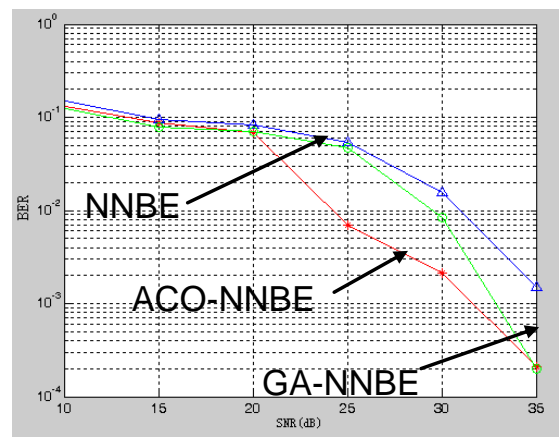

Figure 6. A Comparison of Rate Error Curves in Common Channel 


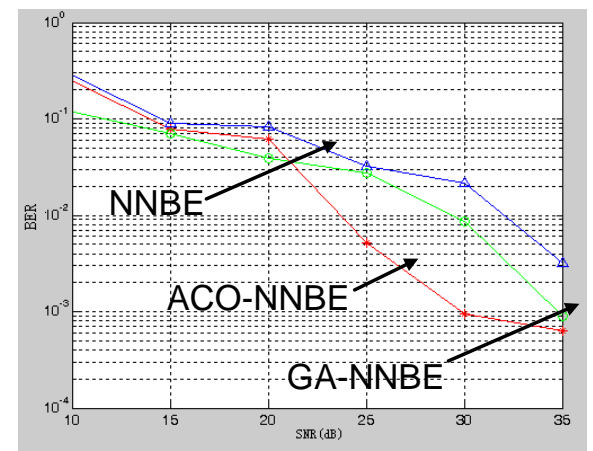

\section{Figure 7. A Comparison of Error Rate Curves in Typical Telephone Channel}

Figure 6 and Figure 7 show the comparison of error rate curves of all algorithms in common channel and in telephone channel respectively. It is observed that ant colony algorithm optimizing neural network in blind equalization algorithm has a lower error rate.

\section{Conclusions}

The thesis combines ant colony optimizing neural network with blind equalization algorithm in order to improve the transmission quality in channels. The randomness and positive feedback of the ant colony algorithm is used to search for the initial weights of neural network. When compared to genetic algorithm optimizing blind equalization algorithm of neural network, ant colony algorithm shows a faster convergence speed and a better convergence performance, which helps to avoid falling into locally optimal solution. In conclusion, the blind equalization of ant colony algorithm optimizing neural network is feasible and effective.

\section{Acknowledgements}

This work is partially supported by National Natural Science Funds of China (No. 61401307); China Postdoctoral Science Foundation (No.2013M530873); Tianjin application foundation and advanced technology research program (13JCYBJC15600); Tianjin application foundation and advanced technology research program (14JCZDJC32600); Tianjin City High School Science \& Technology Fund Planning Project (20130710). The authors also gratefully acknowledge the helpful comments and suggestions of the reviewers, which have improved the presentation.

\section{References}

[1] Hua Wang and Haiqing Cheng, "Modified blind equalization algorithm based on back-propagation neural networks with adaptive momentum factor", Computer Engineering and Design, vol. 5, no. 31, (2010), pp. 1297-1300.

[2] Ying Xiao, Zhenxing Li and Yuhua1 Dong, "Blind Equalization Based on FNN Using Dynamic Object Function”, Journal of System Simulation, vol. 14, no. 21, (2009), pp. 4331-4334.

[3] Haiqing Cheng and Liyi Zhang, "Blind Equal ization Algorithm Using Feed- forward Neural Network Based on a Modified Target Function", Journal of Taiyuan University of Technology, vol. 37, (2006), pp. 39-41.

[4] Donglin Wang and Dongming Wang, "Generalized Derivation of Neural Network Constant Modulus Algorithm for Blind Equalization", Proceedings of 5th International Conference on Wireless Communications, Networking and Mobile Computing (WiCom '09)', Beijing, China, (2009) September 24-26.

[5] Juan Zhao, "Application of the Square Contour Algorithm in Blind Equalizers Based on Complex Neural Networks", Proceedings of 2011 International Conference on Electronics, Communications and Control (ICECC), Ningbo, China, (2013) September 9-11. 
[6] Jiaqi Zhang and Ning Ge, "Joint CMA+ DDLMS blind equalization algorithm", Journal of Tsinghua University (Science and Technology), vol. 49, no. 10, (2009), pp. 108-111.

[7] Wei Xue, Xiaoniu Yang and Zhaoyang Zhang, "T Improved Weighted Multimodulus Blind Equalization Algorithm”, Journal of System Simulation, vol. 23, no. 3, (2011), pp. 451-455.

[8] Wei Bai. The RBF Neural Network Blind Equalization Algorithm Based on Immune Algorithm. Journal of Shanxi Normal University Natural Science Edition. 23, 3 (2011), pp. 451-455.

[9] Zhenxing Liu, Yecai Guo, Min Gao and Xueqing Zhao, "Fuzzy Neural Network Blind Equalization Algorithm Based on Radial Basis Function", Proceedings of 2009 International Conference on Intelligent Human-Machine Systems and Cybernetics (IHMSC 2009), Hangzhou, China, (2009) August 26-27.

[10] Yecai Guo and Lihua Wang, "A Hybrid Wavelet Neural Network Blind Equalization Algorithm Based on Fuzzy Controlling", Acta Electronica Sinica, vol. 39, no. 4, (2011), pp. 975-980.

[11] Yuhua Dong and Junxing Zhang, "Study on Blind Equalization by FNN with Modified Decorrelation Algorithm", Journal of Dalian Nationalities University, vol. 14, no. 5, (2012), pp. 460-462.

[12] Yasong Luo, Jingyuan Lin, Yuxian Hu and Hongning Hu, "Phase Self-amending Blind Equalization Algorithm Using Feedforward Neural Network for High-order QAM Signals in Underwater Acoustic Channels", Journal of Wuhan University of Technology (Transportation Science \& Engineering), vol. 36, no. 6, (2012), pp. 1221-1224.

[13] Yecai Guo, Min Gao and Yanping Zhang, "Feedforward neural network blind equalization algorithm based on orthogonal wavelet packet transform", Journal of Electronic Measurement and Instrument, vol. 23, no. 11, (2009), pp. 59-64.

[14] Zitong Liu, "Research on the Plural Blind Equalization Algorithm Based on Bilinear Feedback Neural Network", Shanxi Electronic Technology, vol.4, (2011), pp. 76-78.

[15] Xiukai Ruan, Chang Li, Yanhua Tan and Yaoju Zhang, "Adaptive Blind Equalization Using Electrical Recurrent Neural Networks", Acta Optica Sinica, vol. 33, no. 5, (2013), pp. 1-12.

[16] Liang Zhang, Rui Chen and Xiaosong Qiu, "Completely automated public turing test to tell computers and humans apart recognition algorithm based on spectral- clustering recurrent neural network ensemble", Journal of Computer Applications, vol. 34, no. 5, (2014), pp. 1383-1385.

[17] Wentie WU, Yuecong Song and Min Li, "Zero Error Nonlinear Correction of Neural Network Optimized by Ant Colony Optimization Algorithm”, Computer Engineering, vol. 34, no. 48, (2012), pp. 97-101.

[18] Liyi Zhang, Ting Liu, Yunshan Sun and Qiang Li, "Research of genetic algorithm optimization neural network blind equalization algorithm based on real number coding", Computer Engineering and Applications, vol. 11, no. 45 (2009), pp. 162-164.

\section{Authors}

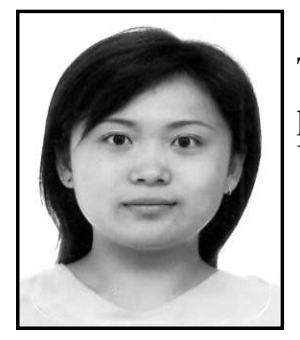

Geng Yanxiang. GENG Yan-xiang (1983-), Female, people of Tianjin city, Postgraduate students, Mainly engaged in information processing research direction of intelligent optimization.Tianjin University of Commerce.Tianjin University.

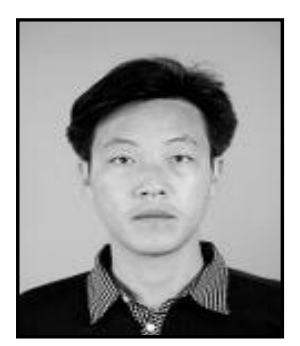

Sun Yunshan. Tianjin University of Commerce.

Tianjin University.

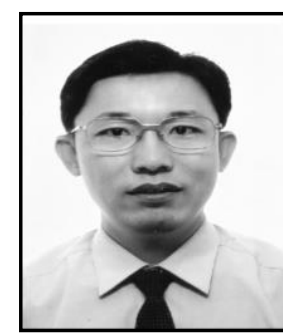

Liyi Zhang. Tianjin University of Commerce.

Tianjin University. 


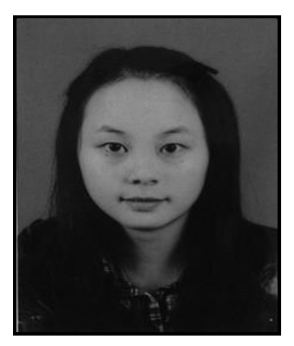

Zhang Yao . Tianjin University of Commerce.

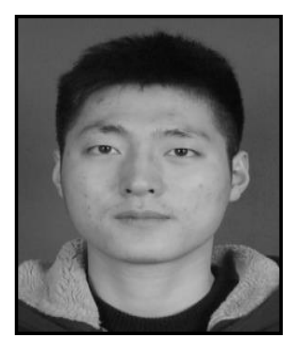

Nan Yang. Tianjin University of Commerce.

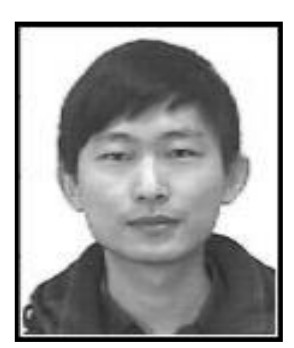

Jiawei Wu. Tianjin University of Commerce. 